\title{
Comportamiento del Semen Bovino Sexado Congelado-Desconge- lado en Fertilización in vitro (FIV) Capacitado Mediante BO en dos Concentraciones versus Percoll
}

\author{
Behavior of Sexed Frozen-Thawed Bovine Semen on in vitro Fertilization (IVF) \\ Capacitated with two Concentrations of BO versus Percoll
}

Filipiak, Y.; Larocca, C. \& Martínez, M.

FILIPIAK, Y.; LAROCCA, C. \& MARTÍNEZ, M. Comportamiento del semen bovino sexado congelado-descongelado en fertilización in vitro (FIV) capacitado mediante BO en dos concentraciones versus Percoll. Int. J. Morphol., 35(4):1337-1341, 2017.

RESUMEN: El objetivo fue comparar la tasa de división (TD) y desarrollo embrionario (DE) con semen sexado X (SX) en FIV

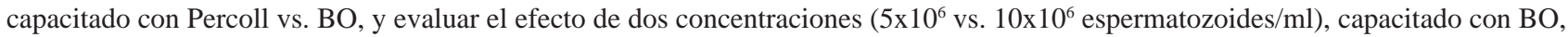
comparado con el semen no-sexado (NS). Un avance importante es predeterminar el sexo en bovinos, es posible obtener mayor proporción de terneras a partir del citómetro de flujo con la capacidad de seleccionar los espermatozoides X por la diferencia del ADN (4 \%, bovinos), con confiabilidad del $90 \%$. El SX aumenta la eficiencia reproductiva, permite la selección de hembras e incrementa la ganancia genética. Se obtuvieron complejos ovocito cúmulus (COC), de ovarios de matadero, se cultivaron para maduración en gotas de 100 $\mu \mathrm{l}$ de TCM-199 + $5 \%$ de suero fetal bovino $+0.005 \mathrm{U} / \mathrm{ml} \mathrm{de} \mathrm{(FSH-p)}+10 \mathrm{IU}$ hCG $/ \mathrm{ml}+1 \mu \mathrm{g}$ Estradiol (E2)/ml, $15 \mathrm{COC} / \mathrm{gota}$, cubiertas con aceite mineral, en incubadora $\left(38,5^{\circ} \mathrm{C}, 5 \%\right.$ de $\mathrm{CO}_{2}$ y $95 \%$ de humedad), 22 h. Posmaduración se dividieron en 4 grupos $(\mathrm{G} 1, \mathrm{G} 2$,

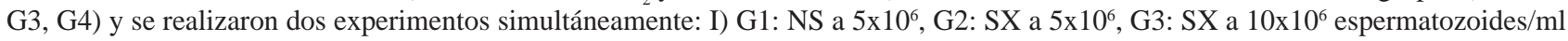
capacitados con BO. II) G2: SX a 4,5x106 espermatozoides/ml capacitados con BO y G4: SX capacitado con Percoll a 5x106 espermatozoides/ $\mathrm{ml}$. Se colocaron $15 \mathrm{COC} /$ gota de semen cubiertas con aceite mineral, en incubadora, $18 \mathrm{~h}$. Para el desarrollo se colocaron en gotas de CR1aa, en incubadora. Se aplicó el test de $\chi^{2}$. La TD a las 48 h entre G1 y G3 no presentó diferencias significativas (p<0,05), sin embargo, en ambos grupos fue significativamente mayor al G2. En el DE al día 7 hubo diferencias significativas $(\mathrm{p}<0,05)$ a favor del G4. Se obtuvo mayor DE con el SX capacitado con Percoll respecto al BO y no hubo diferencias entre ambas concentraciones de semen.

PALABRAS CLAVE: Semen sexado; Fertilización in vitro; Bovinos; Embriones.

\section{INTRODUCCIÓN}

En vaquillonas lecheras el rango de preñez por inseminación artificial (IA) es del $67 \%$ aproximadamente, siendo la relación entre los sexos machos y hembras 50/50. Es de gran importancia lograr predeterminar el sexo de las crías ya que en la cría para leche son de valor las hembras y muy poco valor los machos. Se hicieron muchos intentos en este sentido que no fueron aplicables. La obtención de mayor número de terneras en proporción significativa respecto a los machos se hizo posible a partir de la creación del citómetro de flujo con capacidad de seleccionar los espermatozoides X e Y por la diferencia del ADN (aproximadamente $4 \%$ en bovinos), con una confiabilidad del 90 \% (Xu et al., 2009). Es el único método validado para la selección del sexo precoz actualmente (de Graaf et al., 2009).
Esta tecnología solo se ha desarrollado en el uso comercial en la especie bovina. El uso de semen sexado aumenta la eficiencia reproductiva, permite la selección de hembras superiores para reposición, posibilitando un incremento de la ganancia genética (Weigel, 2004; Mocé et al., 2006; Rath \& Johnson, 2008).

El citómetro de flujo clasifica los espermatozoides, los cuales son cargados por medio de vibración de alta frecuencia en gotas individuales (Schenk et al., 1999). Estos espermatozoides teñidos previamente con bisbenzimidazol (Hoechst 33342) el cual se fija de manera selectiva al ADN (Schenk et al.), generan fluorescencia cuando son sometidos a luz láser y a través de sensores es posible distinguir un

${ }^{1}$ Facultad de Veterinaria. Área Biotecnología de la Reproducción, Departamento de Reproducción Animal.Universidad de la República. Montevideo, Uruguay. 
índice de reflexión superior del espermatozoide $\mathrm{X}$ respecto al espermatozoide Y (Seidel, 2007).

Las gotas son cargadas positiva o negativamente y son desviadas por una placa con la carga opuesta de manera que el esperma es dirigido a un vaso colector para cada una, de forma que los espermatozoides $\mathrm{X}$ van a un vaso colector y los espermatozoides $\mathrm{Y}$ a otro y el desecho a otro (espermatozoides muertos y/o no reconocidos).

Se pueden producir unas 6 a 8 dosis inseminantes, de aproximadamente 2.000 .000 de espermatozoides, por hora, con una eficacia promedio del $90 \%$ del sexo deseado (Ferré \& Cattaneo, 2013).

La baja concentración de espermatozoides en cada dosis de SX (1,5-2 millones/pajuela de $0,25 \mathrm{ml})$ podría ser un factor de la menor tasa de concepción con respecto al uso de NS (Xu et al.) en IA. Para optimizar los resultados, las pajuelas sexadas deberían usarse en vaquillonas que no formen parte de un programa de inseminación a tiempo fijo o en programas de transferencia de embriones (Xu et al.). En IA cuando la dosis de SX es equivalente a la utilizada con semen convencional el rango de preñez para el SX se mantiene en el orden del 60-80 \% de preñez respecto al convencional (Wheeler et al., 2006).

Los primeros terneros nacidos por FIV con SX, nacieron en 1993 (Cran et al., 1993).

A través de la FIV es posible incrementar la eficiencia del SX ya que mientras con una dosis en IA se fertiliza como mucho a una hembra bovina, en FIV es posible fertilizar gran número de ovocitos.

Su aplicación a la producción en combinación con la aspiración folicular in vivo guiada por ultrasonografía (OPU), permitiría obtener mayor cantidad de embriones de alta genética y sexados a menor costo.

Por otro lado, la FIV constituye un modelo experimental para estudiar la fertilidad del SX comercial (Larocca \& Filipiak, 2017).

La capacitación de los espermatozoides en la FIV es substancial para la obtención de buenos índices de producción de embriones. Se han desarrollado diferentes medios de cultivo, de los cuales la centrifugación en gradientes de densidad de Percoll tiene la capacidad de seleccionar los espermatozoides vivos de los muertos, a diferencia de otros medios como el BO (Brackett \& Oliphant, 1975), que los capacita sin seleccionarlos. El medio BO permite la capacitación de los espermatozoides por su contenido de cafeína y heparina y es de bajo costo.
Los gradientes de densidad requieren de una solución (Percoll) diluida en medio de capacitación, cuya densidad aumenta desde la zona superior a la inferior, en la cual el semen es suspendido y al centrifugar, los espermatozoides móviles y con acrosoma intacto migran a través de los gradientes. El Percoll es un medio muy costoso y a veces difícil de obtener, solo permitido su uso veterinario (Palma et al., 2008). La centrifugación en gradiente de Percoll permite que los espermatozoides se desplacen por el gradiente hasta que alcanzan un punto donde su densidad y la del gradiente son idénticas, por lo cual también se le llama centrifugación isopínica. En este punto no se producirá una sedimentación posterior debido a que flotan sobre un "colchón" de material que posee una densidad superior a la suya propia. Como los espermatozoides intactos son de mayor densidad y por lo tanto no son retenidos en los estratos de mejor densidad, estos migran hacia el fondo del tubo, teniendo la ventaja de poder separarlos aun partiendo de muy baja concentración (Palma et al.).

Es de interés evaluar el efecto de diferentes concentraciones de espermatozoides de SX, para definir si la dosis comercial es suficiente para una buena tasa de clivaje y desarrollo en FIV.

Los objetivos de esta investigación fueron: Comparar la fertilidad del SX en FIV capacitado con Percoll vs. BO, medido por la TD y el DE y comparar la fertilidad in vitro de una dosis de $5 \times 10^{6}$ espermatozoides sexados $/ \mathrm{ml}$ vs. $10 \times 10^{6}$ espermatozoides sexados, capacitados con $\mathrm{BO}$ en FIV, medido por la TD y el DE.

\section{MATERIAL Y MÉTODO}

Los COC se obtuvieron de ovarios de hembras bovinas faenadas en mataderos. Fueron transportados en menos de 5 horas en solución salina isotónica a $37^{\circ} \mathrm{C}$. El contenido de los folículos de 2 a $8 \mathrm{~mm}$ de diámetro fue aspirado mediante jeringa con aguja hipodérmica $18 \mathrm{G}$, utilizando medio buffer fosfato salino modificado (m-PBS), con $5 \%$ de suero fetal bovino (SFB) y antibióticos (PenicilinaEstreptomicina). Se utilizaron los COC, clases A y B (normas de la IETS); se lavaron en m-PBS en cajas de Petri de $35 \mathrm{~mm}$ de diámetro y se cultivaron para su maduración en TCM-199 + $5 \%$ de SFB + $0.005 \mathrm{U} / \mathrm{ml}$ de $($ FSH-p $)+10 \mathrm{IU}$ $\mathrm{hCG} / \mathrm{ml}+1 \mu \mathrm{g}$ Estradiol (E2) $/ \mathrm{ml}$, en gotas de $100 \mu \mathrm{l}$ conteniendo 15 COC por gota, cubiertas con aceite mineral, en incubadora $\left(38,5^{\circ} \mathrm{C}, 5 \%\right.$ de $\mathrm{CO}_{2}$ y $95 \%$ de humedad) por 22 horas.

Para la fecundación in vitro se formaron 4 grupos al 
azar con similar número de COC, se realizaron simultáneamente y en las mismas condiciones dos experimentos: Experimento I: El G1 se fecundó con NS a una concentración de $5 \times 10^{6}$ espermatozoides $/ \mathrm{ml}$ (spz/ml), evaluando también el comportamiento in vitro del SX a una concentración de $5 \times 10^{6} \mathrm{spz} / \mathrm{ml}(\mathrm{G} 2)$ y al doble de concentración (G3) midiendo la TD a las 48 horas de la FIV y el DE en el día 7. Experimento II: Se evaluó el comportamiento del SX capacitado con $\mathrm{BO}(\mathrm{G} 2)$ respecto al capacitado con gradientes de Percoll (G4), en ambos diluyendo el semen a $5 \times 10^{6} \mathrm{spz} / \mathrm{ml}$. El G2 está incluido en ambos experimentos ya que al ser SX capacitado con BO, es capaz de enfrentarse tanto al G1 y G3 (Experimento I), pudiendo comparar con el G1 siendo el mismo método de capacitación, pero difiere en que es SX, mientras que el G1 es convencional. También se lo enfrenta con el G3 comparando la diferencia entre las concentraciones espermáticas, siendo el doble en el G3. Para el Experimento II se utiliza el mismo grupo con el fin de comparar los resultados con ambos métodos de capacitación espermática (BO vs. Percoll). En todas las réplicas se usó semen comercial (Nueva Zelanda), congelado-descongelado, del mismo toro y la misma partida.

Las pajuelas de semen $(0,25 \mathrm{ml})$ fueron descongeladas 10 segundos a temperatura ambiente y 30 segundos a 36 ${ }^{\circ} \mathrm{C}$ en baño María. En cada réplica se calculó la concentración inicial mediante cámara de Neubauer. Se estudió la motilidad individual y el vigor al descongelado y a las 2 horas. La capacitación del semen para los grupos G1, G2 y G3 se realizó por centrifugación (500 G), en medio BO de lavado se aspiró el sobrenadante, el pellet que quedó en el fondo del tubo se diluyó con solución BO de dilución ajustando a una concentración de $5 \times 10^{6} \mathrm{spz} / \mathrm{ml}$ para los grupos G1 y G2 y de 10x10 $\mathrm{spz} / \mathrm{ml}$ para el G3, después de estudiar la concentración total, utilizando la cámara de Neubauer. Para el G4 la capacitación se realizó mediante gradientes de Percoll diluidos en Talp-Sperm (90-45 \%). Se centrifugó a
$2500 \mathrm{G}$, por 20 minutos, se aspiró el sobrenadante. Al pellet se le agrego medio Talp-Sperm y se volvió a centrifugar 5 minutos a $2500 \mathrm{G}$, se retiró el sobrenadante y el pellet se ajustó a una concentración de $5 \times 10^{6} \mathrm{spz} / \mathrm{ml}$ con solución Talp-Fert. En platina caliente $\left(36^{\circ} \mathrm{C}\right)$ se formaron gotas de $100 \mu \mathrm{l}$ con el semen, se cubrió con aceite mineral y se colocaron en las gotas los ovocitos madurados, en una relación de 15 COC por gota. El cocultivo se colocó en estufa a 38,5 ${ }^{\circ} \mathrm{C}$, con $5 \%$ de $\mathrm{CO}_{2}$ y $95 \%$ de humedad por un lapso de 18 horas.

Luego de la fecundación, los presuntos cigotos se denudaron mediante vortex en medio TCM-199 HEPES, con $5 \%$ de SFB y antibióticos por 5 minutos, se lavaron y cultivaron para desarrollo en medio CR1aa $+5 \%$ de SFB, en incubadora.

Se estudió la división a las 48 horas (clivaje). A los 7 días a partir de la fecundación se evaluó el desarrollo a embriones transferibles. Para el estudio de los resultados se utilizó el Test de $\chi^{2}$. Los análisis se realizaron con nivel de significación del $5 \%$.

\section{RESULTADOS Y DISCUSIÓN}

La concentración inicial promedio de las pajuelas del SX fue de $2 \times 10^{6} \mathrm{spz} /$ pajuela de $0,25 \mathrm{ml}$, con una motilidad individual promedio del $65 \%$ y 3,5 de vigor (escala 0 a 5 ) y tras 2 horas de incubación a $36{ }^{\circ} \mathrm{C}$ tuvo una motilidad individual de $50 \%$ y 3 de vigor. El SN tuvo una concentración promedio de $14,6 \times 10^{6} \mathrm{esp} /$ pajuela de $0,25 \mathrm{ml}$ y un promedio de motilidad individual $70 \%$ y 4 de vigor. Tras 2 horas de incubación la motilidad individual promedio fue de $60 \%$ y 3,5 de vigor.

Tabla I. TD y DE entre los grupos capacitados con BO (NS vs. SX).

\begin{tabular}{lccccc}
\hline Grupo & $\begin{array}{l}\text { Número total de } \\
\text { COC inseminados }\end{array}$ & \multicolumn{2}{c}{ TD } & \multicolumn{2}{c}{ DE (desarrollo/divididos) } \\
& N & N & $\%$ \\
\hline G2 & 225 & $117 \mathrm{~b}$ & 52,00 & $48^{\mathrm{a}}$ & 41,03 \\
$\mathrm{G} 4$ & 215 & $109 \mathrm{~b}$ & 50,70 & $73^{\mathrm{b}}$ & 66,97 \\
\hline
\end{tabular}

Distintos superíndices difieren significativamente $(\mathrm{p}<0,05)$

Tabla II. TD y DE entre el semen capacitado con Percoll vs. BO.

\begin{tabular}{lccccc}
\hline Grupo & $\begin{array}{l}\text { Número total de COC } \\
\text { inseminados }\end{array}$ & $\mathrm{N}$ & $\%$ & \multicolumn{3}{c}{ DE (desarrollo/divididos) } \\
\hline G1 & 242 & $152^{\mathrm{a}}$ & 62,81 & $50^{\mathrm{a}}$ & 32,89 \\
G2 & 225 & $117 \mathrm{~b}$ & 52,00 & $48^{\mathrm{a}}$ & 41,03 \\
G3 & 158 & $100^{\mathrm{a}}$ & 63,29 & $43^{\mathrm{a}}$ & 43,00 \\
\hline
\end{tabular}

Distintos superíndices difieren significativamente $(\mathrm{p}<0,05)$ 
Los resultados obtenidos en la FIV se muestran en las Tablas I (Experimento I) y II (Experimento II). En el Experimento I, en el clivaje el G1 y el G3 no tuvieron diferencias significativas $(\mathrm{p}<0,05)$ entre sí, en tanto que ambos grupos fue significativamente mayor respecto al G2. En el DE (desarrollo/clivaje) no hubo diferencias significativas entre los tres grupos. Si bien el grupo inseminado con la mayor concentración mostró un mejor clivaje, en el desarrollo obtuvo resultados similares al de menor concentración. En el Experimento II, no hubo diferencias significativas en el clivaje ( $p>0,05)$ entre los grupos G2 y G4. La capacitación del SX con Gradientes de Percoll (G4) arrojó resultados significativamente superiores $(\mathrm{p}<0,05)$ a la capacitación con BO (G2) en el DE.

Nuestros estudios realizados años anteriores (2014) arrojaron mejores resultados en clivaje y DE (Larocca \& Filipiak), el año 2016, en el que se realizaron estos ensayos, en Uruguay fue un año con serios problemas climáticos, abundantes y prolongadas lluvias y como consecuencia falta de alimento para el ganado y deficiente estado corporal, lo cual podría estar vinculado. En estudios anteriores capacitando el NS con BO obtuvimos mayor DE en primaveraverano que en otoño invierno (Filipiak \& Larocca, 2012; Filipiak \& Larocca, 2016).

Los estudios de Xu et al. obtuvieron un clivaje de $61,7 \pm 0 \%$ y un $20,2 \pm 2,5 \%$ de desarrollo con SX en FIV, en nuestra investigación se obtuvieron mayores resultados en DE al utilizar SX.

Se ha sostenido la necesidad de estudios que evalúen el comportamiento in vitro de SX y NS, del mismo toro y en lo posible del mismo eyaculado a la descongelación (Wilson et al., 2006), ya que al estudiar el SX y NS de diferentes toros en FIV no obtuvieron diferencias en la tasa de clivaje, sin embargo, obtuvieron diferencias significativas en la producción de embriones a favor del NS. Resultados similares obtuvieron Trigal et al. (2012) al obtener mayor clivaje y mayor desarrollo de embriones (diferencias significativas), en el NS, respecto al SX. En nuestro trabajo se estudió el comportamiento in vitro del SX y NS del mismo toro y partida, comparando a su vez distintos métodos de capacitación.

Machado et al. (2009) capacitando el SX con gradientes de Percoll obtuvieron con 4 toros diferentes un rango de clivaje de 65 a $85 \%$ y de DE de 17 a $47 \%$, nosotros si bien obtuvimos al usar el Percoll un menor clivaje, el DE fue mayor.

Katska-Ksiaz kiewicz et al. (2006), utilizando SX, semen sexado Y (SY) y NS (congelado-decongelado), reportan diferencias significativas en la tasa de clivaje del NS respecto al semen sexado (X e Y), sin embargo, el DE a blastocistos fue similar para el NS y el SX. Puglisi et al. (2006), estudiando el comportamiento en la FIV de SX y NS provenientes de diferentes toros, reportan una menor tasa de clivaje para el SX, no encontrando diferencias significativas en el DE. Coincidente a los resultados de KatskaKsiazkiewicz et al. y de Puglisi et al., el DE fue similar entre el SX y el NS, salvo al capacitar nosotros el semen con Percoll aumentó significativamente la producción de embriones con SX. Promthep et. al. (2016), utiliza distintos gradientes de Percoll (7 gradientes) con SX y obtiene los mejores resultados en el estudio del semen y en la FIV utilizando el semen obtenido del gradiente con 65 y $70 \%$ de concentración de Percoll. Ellos obtuvieron un $88 \%$ de clivaje y un $34 \%$ de desarrollo, si bien obtuvieron un clivaje mayor, el resultado en embriones es similar a este estudio. La mayoría de los laboratorios de FIV en bovinos, emplean para la capacitación de semen el método de los gradientes de Percoll con semen no sexado y/o sexado.

Un reciente estudio compara tres concentraciones diferentes en FIV, $0,1 \times 10^{6}, 1 \times 10^{6}$ y $10 \times 10^{6} \mathrm{spz} / \mathrm{ml}$ y obtiene menor clivaje y DE en el grupo de mayor concentración de semen. En ese mismo estudio compara también dos métodos de capacitación de semen, el método del Percoll vs. centrifugación simple en Talp-Sperm (sin seleccionar vivos de muertos) y obtuvieron un mayor clivaje y DE con el método del Percoll que selecciona vivos de muertos. Además, encontraron que se producen anomalías cromosómicas cuando el semen está más concentrado, así como cuando no se separa a los espermatozoides muertos (Demyda-Peyrás et al., 2015).

La FIV puede ser una herramienta para producir embriones de sexo deseado en la producción bovina, aumentando los remplazos y disminuyendo el costo relativo del SX al obtenerse mayor cantidad de embriones. La capacitación del SX mediante gradientes de Percoll resulta ser más eficiente en la producción de embriones que el medio $\mathrm{BO}$, sin embargo, su costo es mayor. Aumentar la concentración del semen capacitado con BO no parece ser necesario para obtener mejores resultados en la FIV.

AGRADECIMIENTOS . A Luis Rosés, Dra. Ana Aznares y Dra. Elizabeth Pechiar.

FILIPIAK, Y.; LAROCCA, C. \& MARTÍNEZ, M. Behavior of sexed frozen-thawed bovine semen on in vitro fertilization (ivf) capacitated with two concentrations of BO versus Percoll. Int. J. Morphol., 35(4):1337-1341, 2017.

SUMMARY: The objective of this study was to evaluate the in vitro fertility of bovine sexed semen (SX) capacitated with 
Percoll vs. BO. The division rate (DR), embryo development (ED) were evaluated in two concentrations $5 \times 10^{6}$ vs. $10 \times 10^{6}$ sperm $/ \mathrm{ml}$, capacitated with BO and compared with non-sexed semen (NS). Offspring sexing represents an important advance for livestock production. Flow cytometry separates $\mathrm{X}$ and $\mathrm{Y}$ spermatozoa by difference in DNA content (4\% greater in X) with $90 \%$ effectiveness. The SX increases the reproductive efficiency, allows the selection of females and increases the genetic gain. Cumulusoocytes complexes (COC) were obtained from slaughterhouse ovaries. They were then cultured 22 hours for maturation in TCM$199+5 \%$ fetal calf serum $+0.005 \mathrm{IU} / \mathrm{ml}(\mathrm{FSH}-\mathrm{p})+10 \mathrm{IU} \mathrm{hCG} / \mathrm{ml}$ $+1 \mathrm{mg}$ Estradiol (E2)/ml, in $100 \mu \mathrm{l}$ drops with mineral oil, in incubator $\left(38.5^{\circ} \mathrm{C}, 5 \% \mathrm{CO}\right.$ and $95 \%$ humidity). Post maturation, 4 groups were randomly assigned (G1, G2, G3, G4) and were performed two experiments simultaneously: I) G1 was inseminated with NS at $5 \times 10^{6}$ sperm/ml, G2: SX at $5 \times 10^{6}$, G3: SX at $10 \times 10^{6}$ sperm $/ \mathrm{ml}$ capacitated with BO. II) G2: SX at $5 \times 10^{6} \mathrm{sperm} / \mathrm{ml}$ capacitated with BO and G4: SX capacitated with Percoll at 5x10 sperm $/ \mathrm{ml}$. $15 \mathrm{COC} /$ drop of capacitated semen covered with mineral oil and placed in an incubator for 18 hours. For development, they were placed in drops of CR1aa, in an incubator. Results were analyzed with the $\mathrm{c}$ square test. At 48 hours, there were no significant differences $(\mathrm{p}<0.05)$ in DR between G1 and G3, however, in both groups it was significantly greater than G2. At day 7 there were significant differences $(p<0.05)$ in ED, greater in G4. At 48 hours, there were no significant differences $(\mathrm{p}<0.05)$ in DR between G1 and G3, however, in both groups it was significantly greater than G2. At day 7 there were significant differences $(p<0.05)$ in ED, greater in G4. A higher ED was obtained with the SX capacitated with Percoll, with respect to BO and there was no difference between the two semen concentrations.

KEY WORDS: Sex sorted sperm; in vitro fertilization; Bovine; Embryos.

\section{REFERENCIAS BIBLIOGRÁFICAS}

Brackett, B. G. \& Oliphant, G. Capacitation of rabbit spermatozoa in vitro. Biol. Reprod., 12(2):260-74, 1975.

Cran, D. G.; Johnson, L. A.; Miller, N. G.; Cochrane, D. \& Polge, C. Production of bovine calves following separation of X-and Y-chromosome bearing sperm and in vitro fertilisation. Vet. Rec., 132(2):40-1, 1993.

de Graaf, S. P.; Beilby, K. H.; Underwood, S. L.; Evans, G. \& Maxwell, W. M. Sperm sexing in sheep and cattle: the exception and the rule. Theriogenology, 71(1):89-97, 2009.

Demyda-Peyrás, S.; Dorado, J.; Hidalgo, M. \& Moreno-Millán, M. Influence of sperm fertilising concentration, sperm selection method and sperm capacitation procedure on the incidence of numerical chromosomal abnormalities in IVF early bovine embryos. Reprod. Fertil. Develop., (27):351-9, 2015.

Ferré, L. \& Cattaneo, L. Biotecnologías reproductivas: producción in vitro de embriones y semen sexado. (¿La pareja perfecta?). Rev. Med. Vet. (B. Aires), 94(2):28-36, 2013.

Filipiak, Y. \& Larocca, C.E. Efecto de las Estaciones del Año en los Resultados de la Fertilización In vitro en Bovinos (Bos Taurus) en Zonas Templadas. Casilda, XVII Jornadas de Divulgación Técnico-Científicas 2016, Facultad de Ciencias Veterinarias. Universidad Nacional de Rosario, 2016.

Filipiak, Y. \& Larocca, C.E. Use of trypan blue to determine viability of bovine oocytes for in vitro fertilization. Arch. Zootec., 61(234):309-12, 2012.

Katska-Ksiazkiewicz, L.; Rynska, B.; Bochenek, M.; Opiela, J. \& Jurkiewicz, $\mathrm{J}$. In vitro production of bovine embryos using flow-cytometrically sexed sperm. Arch. Tierz. Dummerstorf., 49(2):133-40, 2006.

Larocca, C. \& Filipiak, Y. In vitro embryo production with frozen-thawed bovine semen sexed. Int. J. Morphol., 35(1):371-5, 2017.

Machado, G. M.; Carvalho, J. O., Filho, E. S.; Caixeta, E. S.; Franco, M. M.; Rumpf, R. \& Dode, M. A. Effect of Percoll volume, duration and force of centrifugation, on in vitro production and sex ratio of bovine embryos. Theriogenology, 71(8):1289-97, 2009.

Mocé, E.; Graham, J. K. \& Schenk, J. L. Effect of sex-sorting on the ability of fresh and cryopreserved bull sperm to undergo an acrosome reaction. Theriogenology, 66(4):929-36, 2006.

Palma, G. A.; Olivier, N. S., Neumüller, Ch. \& Sinowatz, F. Effects of sexsorted spermatozoa on the efficiency of in vitro fertilization and ultrastructure of in vitro produced bovine blastocysts. Anat. Histol. Embryol., 37(1):67-73, 2008.

Promthep, K.; Satitmanwiwat, S.; Kitiyanant, N.; Tantiwattanakul, P.; Jirajaroenrat, K.; Sitthigripong, R. \& Singhapol, C. Practical use of percoll density gradient centrifugation on sperm sex determination in commercial dairy farm in Thailand. Indian J. Anim. Res., 50(3):310-3, 2016.

Puglisi, R.; Vanni, R.; Galli, A.; Balduzzi, D.; Parati, K.; Bognioni, G.; Crotti, G.; Duchi, R.; Galli, C.; Lazzari, G. \& Aleandri, R. In vitro fertilisation with frozen-thawed bovine sperm sexed by flow cytometry and validated for accuracy by real-time PCR. Reproduction, 132(3):519-26, 2006.

Rath, D. \& Johnson, L. A. Application and commercialization of flow cytometrically sex-sorted semen. Reprod. Domest. Anim., 43 Suppl. 2:33846, 2008.

Schenk, J. L.; Suh, T. K.; Cran, D. G. \& Seidel, G. E. Jr. Cryopreservation of flow-sorted bovine spermatozoa. Theriogenology, 52(8):1375-91, 1999.

Seidel, G. E. Jr. Overview of sexing sperm. Theriogenology, 68(3):443-6, 2007.

Trigal, B.; Gómez, E.; Caamaño, J. N.; Muñoz, M.; Moreno, J.; Carrocera, S.; Martín, D. \& Diez, C. In vitro and in vivo quality of bovine embryos in vitro produced with sex-sorted sperm. Theriogenology, 78(7):1465-75, 2012.

Weigel, K. A. Exploring the role of sexed semen in dairy production systems. J. Dairy Sci., 87 Suppl.:E120-30, 2004.

Wheeler, M. B.; Rutledge, J. J.; Fischer-Brown, A.; VanEtten, T.; Malusky, S. $\&$ Beebe, D. J. Application of sexed semen technology to in vitro embryo production in cattle. Theriogenology, 65(1):219-27, 2006.

Wilson, R. D.; Fricke, P. M.; Leibfried-Rutledge, M. L.; Rutledge, J. J.; Penfield, C. M. \& Weigel, K. A. In vitro production of bovine embryos using sex-sorted sperm. Theriogenology, 65(6):1007-15, 2006.

$\mathrm{Xu}$, J.; Chaubal, S. A. \& Du, F. Optimizing IVF with sexed sperm in cattle. Theriogenology, 71(1):39-47, 2009.

\author{
Dirección para Correspondencia \\ Dra. Yael Filipiak \\ Facultad de Veterinaria \\ Área Biotecnología de la Reproducción \\ Departamento de Reproducción Animal \\ Universidad de la República \\ Montevideo \\ URUGUAY
}

\section{E-mail: yael.filipiak@fvet.edu.uy}

Recibido : 22-06-2017

Aceptado: 21-09-2017 